\title{
The Psychiatric Burden on Medical Students in New York City Entering Clinical Clerkships During the COVID-19 Pandemic
}

\author{
Alexandra Saali ${ }^{1} \cdot$ Emma R. Stanislawski $^{1,2}$ - Vedika Kumar ${ }^{1,3} \cdot$ Chi Chan $^{4,5}$. \\ Alicia Hurtado ${ }^{5}$. Robert H Pietrzak ${ }^{6,7}$. Dennis S. Charney ${ }^{5}$. Jonathan Ripp ${ }^{6}$. \\ Craig L. Katz ${ }^{5}$
}

Accepted: 11 September 2021 / Published online: 7 October 2021

(c) The Author(s), under exclusive licence to Springer Science+Business Media, LLC, part of Springer Nature 2021

\begin{abstract}
For medical students first entering the clinical space in July 2020, the unique challenges related to the coronavirus pandemic threatened to amplify the psychological distress associated with clerkship rotations. This study aimed to characterize the mental health of thirdyear medical students starting clinical clerkships in the midst of a pandemic by assessing symptoms of major depressive disorder (MDD), generalized anxiety disorder (GAD), and posttraumatic stress disorder (PTSD) as well as risk, coping, and protective factors associated with psychological outcomes. Of 147 third-year medical students at the Icahn School of Medicine at Mount Sinai in New York City, 110 (75\%) participated in this prospective survey-based study with 108 included in the final analysis. 43 (39.8\%) respondents screened positive for symptoms of either MDD, GAD, or PTSD. Multiple regression analyses revealed that greater overall symptom severity was associated with more avoidant coping, more traumatic events witnessed, poorer student and leisure functioning, lower trait emotional stability, and lower social support. Worries related to COVID-19 did not significantly influence outcome variables. To better understand the role of the pandemic on psychological outcomes in third-year medical students, additional research should focus on the trajectory of these outcomes over the year during the coronavirus pandemic.
\end{abstract}

Keywords Medical education · Coronavirus · Psychological resilience · Depression · Anxiety $\cdot$ Ptsd

\section{Introduction}

Incoming medical students exhibit better mental, emotional and physical health than age-matched peers in the general population [1]. Yet, enrolled medical students experience significantly higher rates of psychological distress than their age-matched

Alexandra Saali and Emma Stanislawki contributed equally to this work and are co-first authors.

Emma R. Stanislawski

emma.stanislawski@gmail.com

Extended author information available on the last page of the article 
counterparts, suggesting that the medical school experience itself may be particularly stressful [2,3]. Pre-COVID-19 pandemic reports of anxiety symptoms among American medical students had anxiety scores at least one standard deviation higher than the general population [2]. Meanwhile, roughly $11 \%$ of the general US adult population reported regular feelings of worry, nervousness or anxiety in 2019 [4]. The overall prevalence of MDD or depressive symptoms among medical students has consistently hovered around $27.2 \%$ for the past 30 years [5], which is at least four times that of the general population (4-5\%) [4].

Traditionally, medical students are educated in the classroom until the third year of medical school, where they rotate through different clinical settings. While adjusting to the clinical wards at the start of that transition, students report the greatest burden of depressive symptoms [6]. Students' perceived level of stress is associated with periods of transition, which may explain why students describe their third year of medical school as the most stressful [7, 8]. Feelings of imposter syndrome, defined as chronic feelings of self-doubt and incompetence despite evidence of abilities, may contribute by precipitating burnout, which has been associated with decreased satisfaction with work-life balance [9]. Moreover, $80 \%$ of students report experiencing a difficult clinical event (such as patient suffering, personal mistreatment by superiors, or poor role modeling) on their rotations, with half of students endorsing that the experience adversely affected their well-being [10]. Specific experiences linked to stress and trauma in the third-year include mistreatment, witnessing patient suffering or death, and encountering poor physician role modeling [11].

Little is known about the experience of medical students in their clinical years during the course of disasters such as pandemics. Previous studies of medical students involved in disaster response have observed an amplified emotional response to a disaster in female students and students participating in intense, less supervised activities [12, 13]. Engagement in relief efforts themselves has not been significantly associated with increased burden of psychiatric illness in the short or long-term [12, 14, 15]. If anything, volunteer participation has been associated with higher levels of post-traumatic growth and professional self-esteem $[12,14]$. On the other hand, in a cohort of New York City medical students in April 2020 during COVID-19 related suspension of clinical rotations, students who volunteered to participate in COVID-19 relief efforts experienced higher ratings of depressive, anxious, and PTSD symptoms than students who did not volunteer [13], suggesting that students' response to the ongoing stresses of the COVID-19 pandemic may be unique.

The novel challenges presented by COVID-19 threaten to amplify the adverse effects of medical school on student mental health $[2,3,16]$. Studies of the general population identified three and four-fold increases in anxious and depressive symptoms from June 2019 to June 2020 [17], with particularly elevated burden in individuals with lower income, exposure to more COVID-19 stressors, single status, age 18-25, performing essential work, and minority racial/ethnic groups $[17,18]$. Chinese medical students reported a high degree of professional pride during the pandemic, while roughly $25 \%$ experienced at least a mild degree of GAD symptoms associated with the financial effects of COVID-19 and delays in academic activities [19, 20]. Similarly, a cross-sectional study in Brazil found high rates of moderate or severe GAD and MDD symptoms in medical students, with a further increase in students who identified as female [21].

During the academic year between June 2020 and June 2021, third-year medical students will be tested by both the clinical experience itself and COVID-19. This study aims to identify the prevalence of MDD, GAD, and PTSD symptoms and the individual factors contributing to this psychological distress in third-year medical students entering the clinical wards in June 2020. 


\section{Methods}

\section{Setting}

This study was conducted at the Icahn School of Medicine at Mount Sinai (ISMMS) in New York City (NYC) between June and July, 2020. 147 students beginning their $3^{\text {rd }}$ year core clerkships were eligible to participate. Students completed rotations in Medicine, Ambulatory Care-Geriatrics Clerkship, Surgery \& Anesthesiology, Obstetrics-Gynecology, Pediatrics, Neurology, and Psychiatry at Mount Sinai health sites across the five boroughs. The study was approved by the institutional review board at the ISMMS (HS\#: 20-00435I GCO\#1: 20-0930(0001) ISMMS).

\section{Design}

All eligible students received an invitation to participate in the survey in June 2020 at their institutional email from a member of the research team. Students were invited to complete the survey via an anonymous electronic platform. In order to receive the \$75 compensation, participants were directed to a Google Form after completing the survey to submit their email addresses in such a way so as not to connect identifying information to their survey responses. The survey took roughly 45 minutes to complete.

\section{Measures}

The survey included validated instruments that measured psychological symptoms, risk factors, coping factors, and protective factors, as well as questions about demographics and worries about the COVID-19 pandemic. Similar measures were used in a 2009 study conducted at the ISMMS that measured the impact of stressful events during the third year of medical students [11]. The measures are as follows:

\section{Mental Health Outcomes}

7-Item Generalized Anxiety Disorder Scale: The GAD-7 assesses minimal, mild, moderate, and severe GAD [22].

8-Item Patient Health Questionnaire: The PHQ-8 is a well-established diagnostic and severity measure for MDD [23].

PTSD Checklist for the DSM-5: The PCL-5 is a widely-used measure of PTSD, corresponding to DSM-5 symptom criteria [24].

\section{Risk Factors}

The Life Events Checklist for the DSM-5: The LEC-5 is a screening survey of traumatic life events that are known to potentially lead to PTSD or distress [25]. Number of items that were endorsed as "Happened to me" were summed as the variable "Number of potentially traumatic events experienced." Number of items that were endorsed as "Witnessed it" were summed as the variable "Number of potentially traumatic events witnessed." 
Childhood Trauma Questionnaire - Short Form: The CTQ-SF is an assessment of a wide range of childhood maltreatment experiences [26].

Social Adjustment Scale, Self report short version: The SAS-SR Short Form measures social functioning in multiple domains [27]. The study used the Student and Leisure sections of the instrument. Higher scores reflect worse functioning.

Worries about COVID-19: At the height of the pandemic, a team of researchers and clinicians with expertise in internal medicine, psychiatry, psychology, and disaster mental health [28] developed 21 questions specifically related to the COVID-19 pandemic. Participants answered on a scale from 1 ("Not worried at all") to 5 ("Worried nearly all the time"). Based on factor analysis, we grouped items into four categories: (1) Worries about equipment, basic needs, and personal consequences, (2) Worries about infecting others, (3) Worries about workload, performance, and schedule, and (4) Worries about getting infected. Factor-based mean scores were then calculated for each category. The internal stability of the scale was high (Cronbach's $\alpha=0.94$ ). Please see supplementary materials for a list of these questions.

\section{Coping Factors}

Brief COPE: The BCOPE Inventory assesses a wide range of common coping strategies grouped into Approach Coping and Avoidant Coping [29].

Religious COPE: The RCOPE Inventory evaluates religious coping in the face of life stressors [30]. Coping strategies were grouped into adaptive coping and maladaptive coping.

\section{Protective Factors}

Connor-Davidson Resilience Scale: The CD-RISC-10 is a 10 item scale that measures resilience, defined as positive adaptation in the face of adversity or trauma [31]. Revised Life Orientation Test: The LOT-R measures dispositional optimism and pessimism [32].

Ten Item Personality Inventory: The TIPI is a brief measure of the Big Five personality dimensions: extraversion, agreeableness, conscientiousness, emotional stability, and openness to experience [33].

Multidimensional Scale of Perceived Social Support: The MSPSS measures three dimensions of social support: family, friend, and significant other [34].

Statistical Analyses (R 4.0.2 (R Core Team, Vienna, Austria) was used for statistical analyses. The four main outcome variables were scores on the PHQ-8, GAD-7, and PCL-5, and a symptom composite score. The symptom composite score was an indicator of general psychiatric distress and was calculated by standardizing and averaging the scores on the PHQ-8, GAD-7, and PCL-5. Missing data on the outcome variables $(<1 \%$ of items in the three screening measures) were imputed using multiple imputation by chained equations [35]. Descriptive statistics were calculated to summarize sample characteristics and symptom severity. Bivariate analyses examined associations between symptom severity and demographic, risk, coping, and protective factors. Nonparametric Spearman's correlations were computed for categorical independent variables and Kruskall-Wallis one-way analyses of variance for continuous independent variables. Four separate multiple linear regression analyses were conducted for PHQ-8, GAD-7, PCL-5, and symptom composite 
scores. For each regression, only independent variables that were significantly associated in the bivariate analysis at $\mathrm{p}<.05$ were included in the multivariate models. Finally, relative importance analyses were conducted to identify the relative proportion of variance that was explained by each of the significant predictors in the multiple linear regressions after accounting for intercorrelations among these predictors [36].

\section{Results}

\section{Participant Characteristics and Prevalence of Psychiatric Symptoms}

Of the 147 third year medical students who were invited to participate, 110 (75\%) completed the survey. Of the 110 respondents, two had missing data on all items of the screening measures that could not be imputed, resulting in a final sample of 108 students. Tables 1 and 2 present participant characteristics and symptom severity among the sample. 43 (39.8\%) respondents screened positive for symptoms of either MDD, GAD, or PTSD, with positive screens for GAD, MDD and PTSD in 32\%, 24\% and 7\% of students respectively.

\section{Correlates of GAD, MDD, PTSD and Symptom Composite Score}

Table 3 presents the bivariate associations between the symptom composite score and scores on the PHQ-8, GAD-7, PCL-5 with demographic, risk, coping, and protective factors.

\section{Psychiatric Symptom Composite Scores}

Only those factors that were significantly associated with the outcome variables in the bivariate analyses were entered into their respective multiple linear regressions. Results of the multiple linear regressions are presented in Table 4. The model predicting overall symptom severity accounted for $60 \%$ of the variance in symptom composite score. Greater symptom composite scores were significantly associated with greater number of potentially traumatic experiences witnessed (LEC-5), poorer social functioning (SAS-SR), increased use of avoidance as a coping strategy (BCOPE), lower trait emotional stability (i.e, less calm, easily upset, anxious; TIPI), and lower social support (MSPSS). Relative importance analysis revealed that the majority of the total variance explained in the symptom composite was accounted for by avoidant coping (18.0\% of total variance explained), trait emotional stability (15.1\%), and social functioning (13.7\%), compared with traumatic experiences witnessed and self-reported social support accounting for only $9.4 \%$ and $9.0 \%$, respectively.

\section{MDD, GAD, and PTSD Symptoms}

Greater symptoms of MDD were associated with increased avoidant coping (accounting for $19.2 \%$ of total variance explained) and lower trait emotional stability (26.5\% of total variance explained) in the relative importance analysis. The overall model accounted for $41 \%$ of the variance in the PHQ- 8 total score. Greater symptoms of PTSD were 
Table 1 Sample characteristics of n=108 ISMMS medical students in Summer 2020

$n(\%)$ or mean $(\mathrm{SD})$

Demographics

Age

Gender, female

Ethnicity

Caucasian

Asian

Other

Relationship status, single

Seeking dual degree

\section{Risk Factors}

No. of potentially traumatic events experienced (LEC-5)

No. of potentially traumatic events witnessed (LEC-5)

Childhood trauma (CTQ)

Student and leisure functioning (SAS-SF)

Worries about equipment, basic needs, and consequences

$1.9(0.7)$

$2.5(0.9)$

$3.3(1.0)$

$3.4(1.0)$

Worries about workload, performance, and schedule

2.5 (1.0)

\section{Coping}

Approach coping (BCOPE)

$32.0(7.7)$

Avoidant coping (BCOPE)

Adaptive religious coping (RCOPE)

Maladaptive religious coping (RCOPE)

\section{Protective Factors}

Conscientious trait (TIPI)

Emotional stability trait (TIPI)

Extraversion trait (TIPI)

Agreeableness trait (TIPI)

Openness to experience trait (TIPI)

Resilience (CD-RISC)

$28.6(6.4)$

Optimism (LOT-R)

$14.1(4.4)$

Social support (MSPSS)
$5.6(1.1)$

LEC-5 Life Events Checklist for DSM-5, CTQ Childhood Trauma Questionnaire, SAS-SF Social Adjustment Scale-Self-Report, $B C O P E$ Brief COPE, RCOPE Brief RCOPE, TIPI Ten-Item Personality Inventory, $C D$-RISC Connor-Davidson Resilience Scale, LOT-R Life Orientation Test-Revised, MSPSS Multidimensional Scale of Perceived Social Support

associated with greater number of potentially traumatic events witnessed (accounting for $22.2 \%$ of total variance explained), poorer social functioning (16.5\% of total variance explained), more avoidant coping (13.3\% of total variance explained), and less social support (16.5\% of total variance explained). The overall model accounted for $54 \%$ of the variance in PCL-5 total score. None of the predictor variables were significant in the multiple regression model for severity of GAD symptoms. 
Table 2 Psychiatric symptoms among $n=108$ ISMMS medical students in Summer 2020 $n(\%)$ or mean $(\mathrm{SD})$

\section{Depression}

\begin{tabular}{ll}
\hline PHQ-8 score, mean (SD) & $5.83(5.43)$ \\
PHQ-8 symptom severity, n (\%) & $56(51.9)$ \\
None & $26(24.1)$ \\
mild & $17(15.7)$ \\
moderate & $7(6.5)$ \\
$\quad$ moderately severe & $2(1.9)$ \\
$\quad$ severe & \\
Anxiety & $7.36(5.79)$ \\
GAD-7 score, mean (SD) & \\
GAD-7 symptom severity, n (\%) & $42(38.9)$ \\
minimal & $31(28.7)$ \\
mild & $20(18.5)$ \\
moderate & $15(13.9)$ \\
$\quad$ severe & \\
PTSD & $11.86(12.81)$ \\
PCL-5 score, mean (SD) & \\
\hline PHQ-8 8-item Patient Health Questionnaire, GAD-7 7-item General- \\
ized Anxiety Disorder Scale, PCL-5 Posttraumatic Stress Disorder \\
(PTSD) Checklist for DSM-5
\end{tabular}

\section{Discussion}

The intense pressures and demands of medical training are especially salient during the clerkship years and can lead to detrimental psychological outcomes [11, 37, 38]. Over the 2020-2021 academic year, ISMMS students rotating on the wards in NYC had to contend not only with the stress of clinical immersion, but also unique challenges presented by COVID-19. In this study, conducted at the start of the third year, we found that $40 \%$ of students at the ISMMS screened positive for at least one of the following psychiatric conditions: GAD, MDD or PTSD. Psychiatric burden was higher in students with avoidant coping styles, lower trait emotional stability, lower social support and social functioning, and those who had witnessed a greater number of traumatic events.

In a 2009 mental health surveys of third-year ISMMS students, $4 \%$ of students screened positive for GAD (measured by the Beck Anxiety Index), $6 \%$ of students screened positive for MDD (measured by the Beck Depression Index), and 0\% of students screened positive for PTSD. ${ }^{11}$ In comparison, the results of our June 2020 study illustrate an increase in psychiatric burden amongst medical students, with positive screens for GAD, MDD and PTSD in $32 \%, 24 \%$ and $7 \%$ of students respectively.

The prevalence of positive screens of MDD and GAD identified in this cohort were similar to those reported in frontline healthcare workers in Spring 2020 during the height of the pandemic [28, 39] and paralleled the general population's mental health in June 2020 [17]. As a result, it would be reasonable to assume the psychological impacts of COVID-19 contributed substantially to the increase in psychiatric burden amongst ISMMS students. Surprisingly, worries about COVID-19 were not significant in the models predicting well-being outcomes. Participants may have been influenced by 


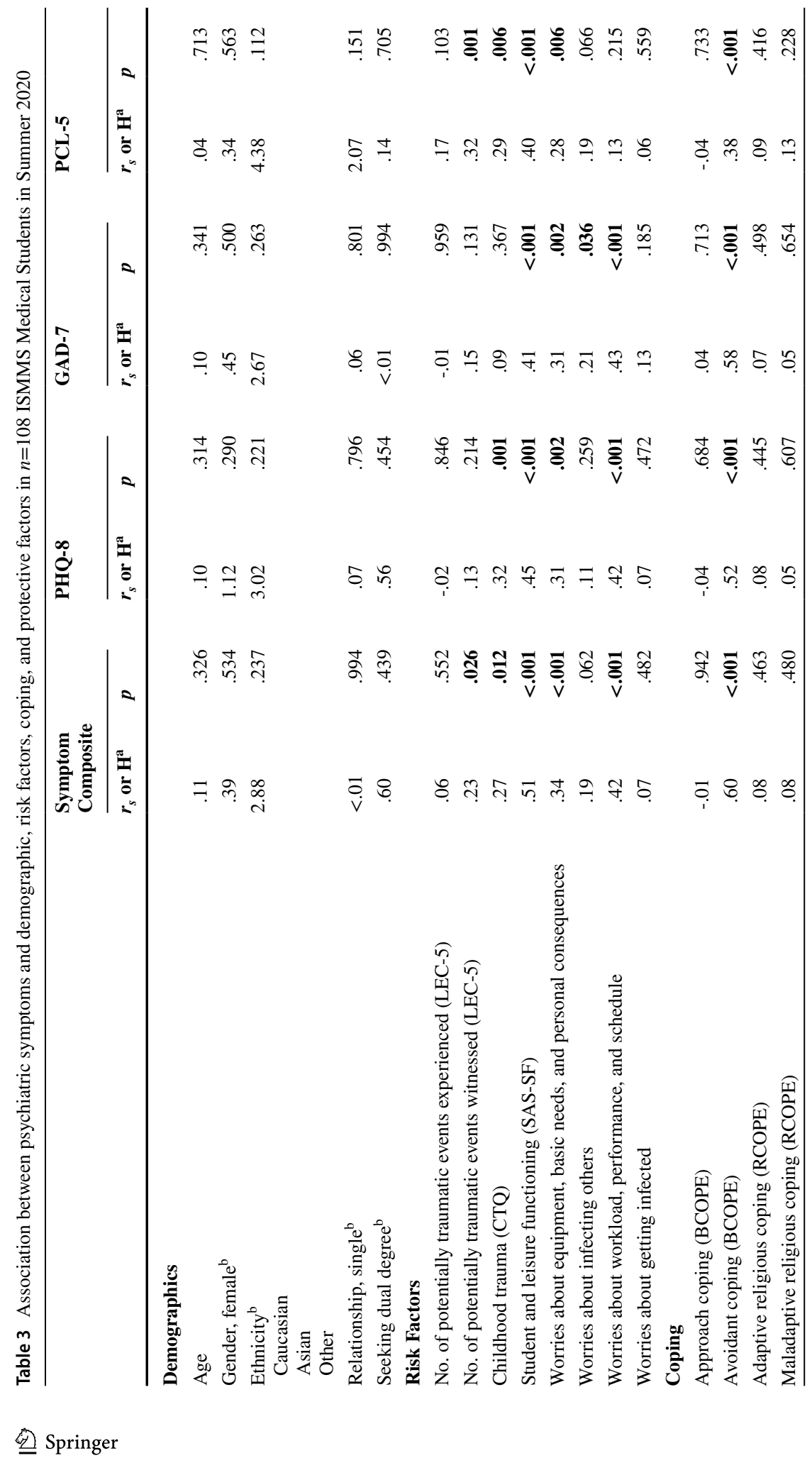




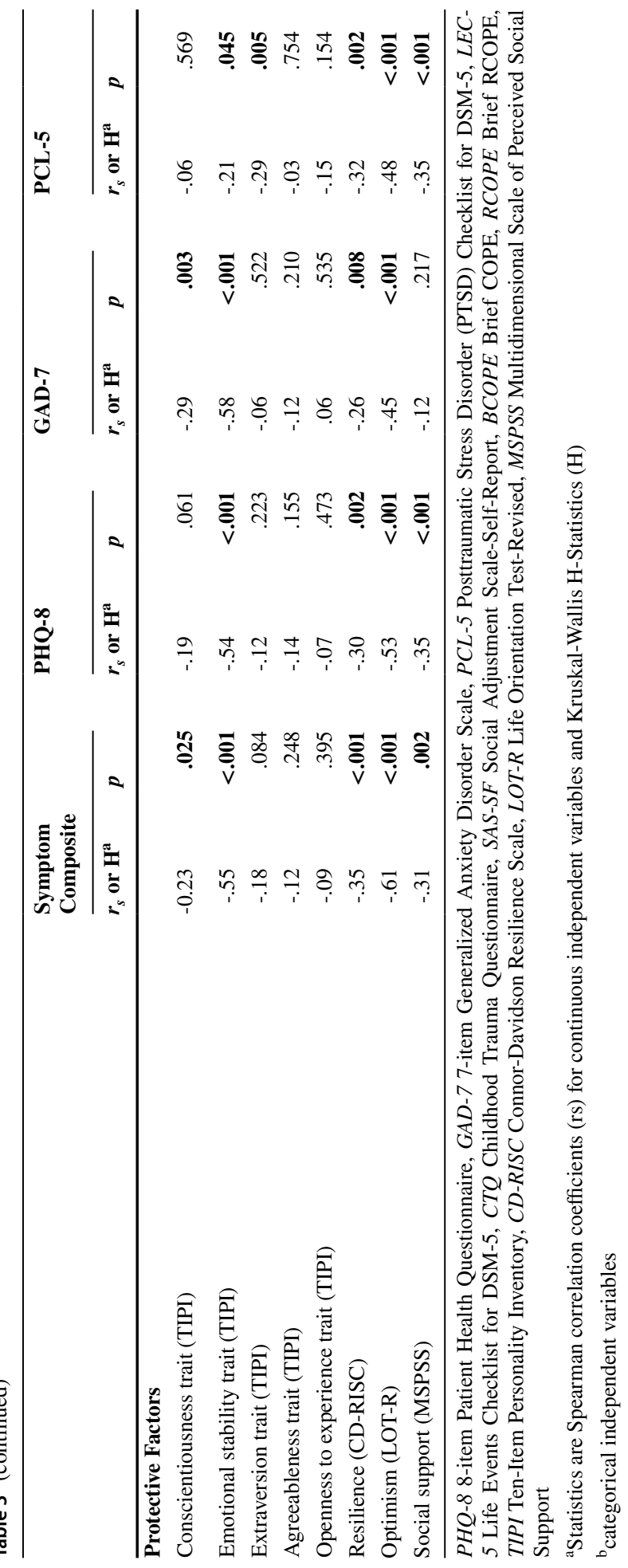




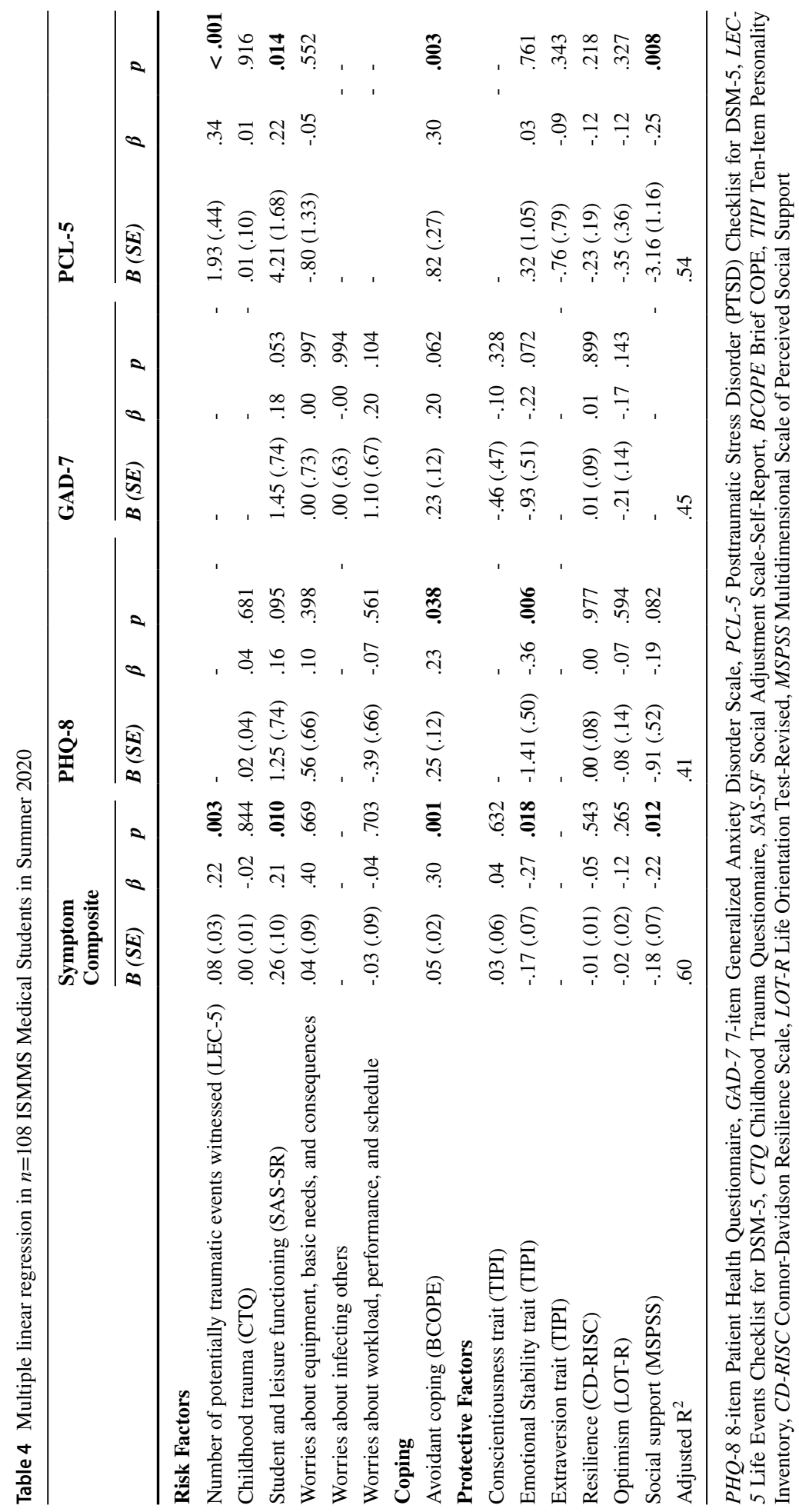


declining cases, hospitalizations and deaths related to COVID-19 in NYC at the time of survey distribution [40]. Additionally, many students were still quarantined off campus. Therefore, worries about COVID-19 may not have yet affected this cohort of students as strongly as front-line workers or even student volunteers [13, 41, 42].

Yet, COVID-19 may have complicated, amplified or forced students to confront other sources of distress, exerting indirect influence on their psychological well-being. While the questions we asked about COVID-19 worries touched on clinical, academic, and health concerns, these questions are not exhaustive. As a result, it might not have captured the full scope of the pandemic's impact on student life. Additionally, during this period of time, the murders of George Floyd, Breonna Taylor, Ahmaud Arbery and other Black Americans highlighted the systemic racism in the United States, and sparked protests against police brutality across the globe. Third-year students were also wrestling with academic concerns, including Step 1 scheduling disruptions and changes to the classical structure of the third year. Using the Twitter "Hedonometer", a tool that tracks public sentiment by sampling $10 \%$ of tweets each day and categorizes them as positive or negative, scientists reported the two week stretch from May 26th-June 9th 2020 as the saddest two weeks on Twitter [43]. Our study collected data on the tail end of this time point, suggesting that early summer of 2020 was a particularly challenging moment in time. Future studies should seek to understand not only the impact of COVID-19, but also how personal, political and current events played a role in the mental health of students during 2020 and beyond.

Our current findings can be used by educational leaders to inform medical student wellbeing and mental health interventions. Our results, along with more recent reports of MDD and GAD amongst students surveyed in the last several years, demonstrate a mental health crisis amongst trainees, irrespective of these acute stressors such as COVID-19 [5, 44, 45]. Given the relationship we found between avoidant coping mechanisms and psychiatric burden, programming that educates students about the value of confronting difficult experiences and emotions may be beneficial. As students benefit most from discussing trauma with their teammates [11], school leaders might also consider training peer or residenteducators in evidence-based traumatic stress processing interventions [46]. Importantly, students who employ avoidant coping strategies may not be inclined to speak up in a room, seek out support from designated advocates, nor alert someone of a traumatic event. With this vulnerable population in mind, more proactive well-being screening and delivery of interventions by medical institutions that do not rely solely on students' self reports or requests for help may be beneficial. Additionally, given the finding that lower social support and poorer social functioning is associated with increased psychiatric distress, medical educators could consider building social and leisure activities into curricula to encourage rest and socialization. By taking these actions for students, institutional leadership can demonstrate its support, which is closely linked to improved psychological well-being amongst front-line healthcare workers [41].

\section{Limitations}

There are several limitations to this study. The cross-sectional design limits our ability to infer causal relationships. All of our survey instruments were self-reported and survey based, therefore subject to response bias. Due to the stigma around mental illness, respondents may have been reluctant to disclose their symptoms. The instruments asked students to look back on past events and symptoms, leading to the possibility of recall bias. Our school-wide study may not generalize to students around the country at other schools. 
This is particularly possible given COVID-19's differential impact on various parts of the United States and the variable timeline of COVID-19 surges across the country.

\section{Future Directions}

The physical and mental health of our front-line providers and trainees plays a pivotal role in the fight against COVID-19. To this end, medical institutions are monitoring physical symptoms of COVID-19 in students and employees. The prevalence of psychological distress in medical students seen in this study suggests that medical institutions should similarly be tracking and responding to symptoms of psychological distress. We plan to administer quarterly mental health surveys to third year students at the ISMMS over the 2020-2021 academic year to observe the interaction of the stress of clinical rotations, personal life events, and the pandemic.

\section{Conclusions}

The results of the present study demonstrate that a large proportion of third year medical students entering the clinical wards during the COVID-19 pandemic in NYC experienced significant psychological burden. In this study, coping skills, personality traits, previous traumas, and social interactions largely explained this psychological distress. Concerns regarding COVID-19 did not appear to meaningfully contribute, possibly because students had not yet entered the clinical wards at the time of data collection. These findings suggest that developing healthy coping-skills, fostering interpersonal supportive relationships, and prioritizing leisure time in the clinical year may be useful interventions for medical educators to develop and incorporate into their curricula.

Supplementary Information The online version contains supplementary material available at https://doi. org/10.1007/s11126-021-09955-2.

Acknowledgements The authors wish to thank Kira Schidmtfor her administrative efforts, as well as Dr. Nihal Mohamed, Dr. David Muller and the Medical Education team at the Icahn School of Medicine at Mount Sinai for their thoughtful feedback on this work.

Author Contributions Alexandra Saali and Emma Stanislawski are co-first authors on this paper. Both shared equal roles in the conceptualization and design of the study, data collection, writing and editing the manuscript, and project administration. Vedika Kumar was involved in conceptualization, design, data collection, and writing and editing of the manuscript. Chi Chan was involved in data analysis and visualization, as well as reviewing and editing the manuscript. Alicia Hurtado was involved in the conceptualization and design of the study as well as reviewing and editing the manuscript. Robert H Pietrzak was involved in the data analysis as well as reviewing and editing the manuscript. Dennis S. Charney was involved in the conceptualization as well as reviewing and editing the manuscript. Jonathan Ripp was involved in the conceptualization and design of the study as well as reviewing and editing the manuscript. Craig L. Katz was involved in the conceptualization and design of the study as well as the material preparation, data collection, and reviewing and editing the manuscript.

Funding This study was funded by the Office of Well-Being and Resilience (OWBR) at the Icahn School of Medicine at Mount Sinai. Dr. Ripp and Dr. Charney are members of the OWBR and had a role in the design of the data and writing of the report.

Code Availability R 4.0.2 was used for statistical analyses. No custom code is available. 


\section{Declarations}

Ethics Approval The study was approved by the Institutional Review Board (IRB) at the Icahn School of Medicine at Mount Sinai in May 2020 and determined to be exempt with a waiver of informed consent (HS\#: 20-00435| GCO\#1:20-0930(0001) ISMMS).

Consent to Participate/Publication This study was deemed by the IRB at ISMMS to have a waiver of informed consent. All participants accessed a form at the beginning of each survey informing them that their responses were collected for use in a research study. No identifying information was collected for this study.

Conflicts of Interest Dr. Charney is named as co-inventor on patents filed by the Icahn School of Medicine at Mount Sinai (ISMMS) relating to the treatment for treatment-resistant depression, suicidal ideation and other disorders. ISMMS has entered into a licensing agreement with Janssen Pharmaceuticals, Inc. and it has and will receive payments from Janssen under the license agreement related to these patents for the treatment of treatment-resistant depression and suicidal ideation. Consistent with the ISMMS Faculty Handbook (the medical school policy), Dr. Charney is entitled to a portion of the payments received by the ISMMS. Since SPRAVATO has received regulatory approval for treatment-resistant depression, ISMMS and thus, through the ISMMS, Dr. Charney, will be entitled to additional payments, beyond those already received, under the license agreement. Dr. Charney is a named co-inventor on several patents filed by ISMMS for a cognitive training intervention to treat depression and related psychiatric disorders. The ISMMS has entered into a licensing agreement with Click Therapeutics, Inc. and has and will receive payments related to the use of this cognitive training intervention for the treatment of psychiatric disorders. In accordance with the ISMMS Faculty Handbook, Dr. Charney has received a portion of these payments and is entitled to a portion of any additional payments that the medical school might receive from this license with Click Therapeutics. Dr. Charney is a named co-inventor on a patent application filed by the ISMMS for the use of intranasally administered Neuropeptide Y (NPY) for the treatment of mood and anxiety disorders. This intellectual property has not been licensed. Dr. Charney is a named co-inventor on a patent application in the US, and several issued patents outside the US filed by the ISMMS related to the use of ketamine for the treatment of post-traumatic stress disorder (PTSD). This intellectual property has not been licensed. No other declarations of interest to disclose.

\section{References}

1. Brazeau CM, Shanafelt T, Durning SJ, et al. Distress among matriculating medical students relative to the general population. Acad Med. 2014;89(11):1520-5.

2. Dyrbye LN, Thomas MR, Shanafelt TD. Systematic review of depression, anxiety, and other indicators of psychological distress among U.S. and Canadian medical students. Acad Med.2006;81(4):354-73.

3. Slavin SJ. Medical student mental health: Culture, environment, and the need for change. JAMA. 2016;316(21):2195-6.

4. Clarke T, Boersman P, Schiller J. Early Release of Selected Estimates Based on Data From the 2019 National Health Interview Survey. Anxiety and Depression Association of America, ADAA. 2019. https://adaa.org/understanding-anxiety/depression (Accessed 4 Jan 2021).

5. Rotenstein LS, Ramos MA, Torre M, et al. Prevalence of depression, depressive symptoms, and suicidal ideation among medical students: A systematic review and meta-analysis. JAMA. 2016;316(21):2214-36.

6. Paro HB, Morales NM, Silva CH, et al. Health-related quality of life of medical students. Med Educ. 2010;44(3):227-35.

7. Lin YK, Lin CD, Lin BJ, et al. Medical students' resilience: A protective role on stress and quality of life in clerkship. BMC Med Educ. 2019;19:473.

8. Radcliffe C, Lester H. Perceived stress during undergraduate medical training: A qualitative study. Med Educ. 2003;37(1):32-8.

9. Shanafelt T, Hasan O, Dyrbye LN, et al. Changes in burnout and satisfaction with work-life balance in physicians and the general US working population between 2011 and 2014. Mayo Clin Proc. 2015;90(12):1600-13.

10. Houpy JC, Lee WW, Woodruff JN, Pincavage AT. Medical student resilience and stressful clinical events during clinical training. Med Educ Online. 2017;22(1):1320187. 
11. Haglund ME, aan het Rot M., Cooper N.S., et al. Resilience in the third year of medical school: A prospective study of the associations between stressful events occurring during clinical rotations and student well-being. Acad Med. 2009;84(2):258-68.

12. Katz CL, Gluck N, Maurizio A, DeLisi LE. The medical student experience with disasters and disaster response. CNS Spectr. 2002;7(8):604-10.

13. Stanislawski ER, Kumar V, Katz CL. PsychologicaliImpact of COVID-19 on medical students in New York City. Poster presented at american academy of child and adolescent psychiatry's virtual annual meeting. Online. 2020.

14. Anderson D, Prioleau P, Taku K, et al. Post-traumatic stress and growth among medical student volunteers after the March 2011 disaster in Fukushima, Japan: Implications for student involvement with future disasters. Psychiatr Q. 2016;87(2):241-51.

15. Nabors C, Frishman WH, Dhand A, et al. The impact on medical students of the $9 / 11$ attacks on New York's world trade center. Teach Learn Med. 2020;1-10.

16. O'Byrne L, Gavin B, McNicholas F. Medical students and COVID-19: The need for pandemic preparedness. J Med Ethics. 2020;46(9):623-6.

17. Czeisler MÉ, Lane RI, Petrosky E, et al. Mental health, substance use, and suicidal ideation during the COVID-19 pandemic — United States, June 24-30, 2020. MMWR Morb Mortal Wkly Rep. 2020;69:1049-57.

18. Ettman CK, Abdalla SM, Cohen GH, Sampson L, Vivier PM, Galea S. Prevalence of depression symptoms in US adults before and during the COVID-19 pandemic. JAMA Netw Open. 2020;3(9):e2019686.

19. Cao W, Fang Z, Hou G, et al. The psychological impact of the COVID-19 epidemic on college students in China. Psychiatry Res. 2020;112934.

20. Zhan J, Sun S, Xie L, Wen Y, Fu J. Medical students' mental health, professional pride, and intention to work in the front-line during coronavirus disease 2019 pandemic. J Cent South Univ. 2020;45(6):649-56.

21. Sartorao Filho CI, Rodrigues WC, de Castro RB, et al. Impact Of covid-19 pandemic on mental health of medical students: A cross-sectional study using GAD-7 and PHQ-9 questionnaires. MedRxiv. 2020.

22. Löwe B, Decker O, Müller S, et al. Validation and standardization of the generalized anxiety disorder screener (GAD-7) in the general population. Med Care. 2008;266-74.

23. Kroenke K, Strine TW, Spitzer RL, et al. The PHQ-8 as a measure of current depression in the general population. J Affect Disord. 2009;114(1-3):163-73.

24. Blevins CA, Weathers FW, Davis MT, Witte TK, Domino JL. The posttraumatic stress disorder checklist for DSM-5 (PCL-5): Development and initial psychometric evaluation. J Trauma Stress. 2015;28(6):489-98.

25. Weathers FW, Blake DD, Schnurr PP, Kaloupek DG, Marx BP, Keane TM. The life events checklist for DSM-5 (LEC-5). Instrument available from the National Center for PTSD at www.ptsd.va.gov. 2013.

26. Bernstein DP, Stein JA, Newcomb MD, et al. Development and validation of a brief screening version of the childhood trauma questionnaire. Child Abuse Neglect. 2003;27(2):169-90.

27. Gameroff MJ, Wickramaratne P, Weissman MM. Testing the short and screener versions of the social adjustment scale-self-report (SAS-SR). Int J Meth Psych Res. 2012;21(1):52-65.

28. Feingold JH, Peccoralo L, Chan CC, et al. Psychological impact of the COVID-19 pandemic on frontline health care workers during the pandemic surge in New York City. Chronic Stress. 2021;5:2470547020977891.

29. Carver CS. You want to measure coping but your protocol's too long: consider the brief COPE. Int J Behav Med. 1997;4(1):92-100. https://doi.org/10.1207/s15327558ijbm0401_6.

30. Pargament K, Feuille M, Burdzy D. The Brief RCOPE: Current psychometric status of a short measure of religious coping. Religions. 2011;2(1):51-76.

31. Campbell-Sills L, Stein MB. Psychometric analysis and refinement of the connor-davidson resilience scale (CD-RISC): Validation of a 10-item measure of resilience. J Trauma Stress. 2007;20(6):1019-28.

32. Hinz A, Sander C, Glaesmer H, et al. Optimism and pessimism in the general population: Psychometric properties of the Life Orientation Test (LOT-R). Int J Clin Hlth Psyc. 2017;17(2):161-70.

33. Gosling SD, Rentfrow PJ, Swann WB Jr. A very brief measure of the big-five personality domains. J Res Pers. 2003;37(6):504-28.

34. Kazarian SS, McCabe SB. Dimensions of social support in the MSPSS: Factorial structure, reliability, and theoretical implications. J Community Psychol. 1991;19(2):150-60.

35. Azur MJ, Stuart EA, Frangakis C, Leaf PJ. Multiple imputation by chained equations: what is it and how does it work? Int J Meth Psych Res. 2011;20(1):40-9.

36. Grömping U. Relative importance for linear regression in R: The package relaimpo. J Stat Softw. 2006;17(1):1-27. 
37. Roberts LW, Warner TD, Lyketsos C, et al. Perceptions of academic vulnerability associated with personal illness: A study of 1,027 students at nine medical schools. Compr Psychiatry. 2001;42(1):1-15.

38. Schwenk TL, Davis L, Wimsatt LA. Depression, stigma, and suicidal ideation in medical students. JAMA. 2010;304(11):1181-90.

39. Sheraton M, Deo N, Dutt T, et al. Psychological effects of the COVID 19 pandemic on healthcare workers globally: A systematic review. Psychiatry Res. 2020;292:113360.

40. New York City COVID Data Trends. NYC.gov website. 2020. https://www1.nyc.gov/site/doh/covid/ covid-19-data-trends.page (Accessed 15 Jan 2021).

41. Pietrzak RH, Feingold JH, Feder A, et al. Psychological resilience in frontline healthcare workers during the acute phase of the COVID-19 pandemic in New York City. J Clin Psychiat. 2020;82(1):20113749.

42. Shanafelt T, Ripp J, Trockel M. Understanding and addressing sources of anxiety among health care professionals during the COVID-19 pandemic. JAMA. 2020;323(21):2133-4.

43. Has Twitter just had its saddest fortnight ever? Nature News. 2020. https://www.nature.com/articles/ d41586-020-01818-3. (Accessed 15 June 2020).

44. Liu CH, Zhang E, Wong GTF, et al. Factors associated with depression, anxiety, and PTSD symptomatology during the COVID-19 pandemic: Clinical implications for U.S. young adult mental health. Psychiat Res. 2020;290.

45. Puthran R, Zhang MW, Tam WW, et al. Prevalence of depression amongst medical students: A metaanalysis. Med Educ. 2016;50(4):456-68.

46. Mitchell JT. When disaster strikes. JEMS-J Emerg Med Ser. 1983;8:36-9.

Publisher's Note Springer Nature remains neutral with regard to jurisdictional claims in published maps and institutional affiliations.

Alexandra Saali, BA studies at the Icahn School of Medicine at Mt. Sinai in NYC (MS4), where she leads the student-run free mental health clinic (MHC) at the East Harlem Health Outreach Partnership (recipient of the APA 2020 Award for Advancing Minority Mental Health). As the chair of the MHC and a studentprovider she has piloted cognitive behavioral therapy, psychodynamic psychotherapy, an AUD buddy program, outpatient $\mathrm{C} / \mathrm{L}$ and telepsychiatry services. Alexandra is a member of the Gold Humanism Honor Society, Mt. Sinai sole nominee for the AMA Physicians of Tomorrow: Peck Health Equity Scholarship, and the recipient of the student body 2021 Excellence in Mentoring Award, commendation for outstanding service as well as commendation for incredible response to COVID-19.

Emma Stanislawski, MD is a first year psychiatry resident at Maine Medical Center. She completed medical school at the Icahn School of Medicine at Mount Sinai. Her research interests include medical education and the intersection of technology and psychiatry.

Vedika Kumar, MD is a first year psychiatry resident at Zucker Hillside Hospital. She completed medical school at the Icahn School of Medicine at Mount Sinai.

Chi Chan, PhD is Assistant Professor of Psychiatry at Icahn School of Medicine at Mount Sinai and Research Health Scientist at the James J. Peters VA Medical Center. Her research focuses on understanding the cognitive processes and corresponding neural mechanisms that are associated with severe mental illness.

Alicia Hurtado, MD is an assistant professor in the Department of Medical Education and Psychiatry, Icahn School of Medicine at Mount Sinai, New York, New York where she serves as the Associate Dean for Medical Student Wellbeing and Student Affairs. She also serves as an attending in the department of psychiatry where she directly supervises psychiatry residents in the outpatient setting.

Robert H. Pietrzak, MPH, PhD is Director of the Translational Psychiatric Epidemiology Laboratory in the Clinical Neurosciences Division of the U.S. Department of Veterans Affairs National Center for PTSD and Professor of Psychiatry at Yale University School of Medicine and Professor of Public Health (Social and Behavioral Sciences) at Yale School of Public Health. His primary research interests include the epidemiology of traumatic stress and resilience across the lifespan; dimensional models of stress-related psychopathology; and the effect of stress on cognition. 
Dennis S. Charney, MD is Anne and Joel Ehrenkranz Dean of the Icahn School of Medicine at Mount Sinai and President for Academic Affairs for the Mount Sinai Health System. He is a world-renowned expert in the neurobiology and treatment of mood and anxiety disorders who has made fundamental contributions to the understanding of human anxiety, fear, depression, and resilience, and has played a key role in the discovery of new treatments for mood and anxiety disorders.

Jonathan Ripp, MD, MPH is Professor of Medicine, Medical Education and Geriatrics and Palliative Medicine, Senior Associate Dean for Well-Being and Resilience and Chief Wellness Officer at the Icahn School of Medicine at Mount Sinai (ISMMS). In the role of chief wellness officer, Dr. Ripp oversees efforts to assess and provide direction for system- and individual-level interventions designed to improve well-being for all students, residents, fellows and faculty in the Mount Sinai Health System. Dr. Ripp also co-founded and is the former Director of the ISMMS Department of Medicine's Advancing Idealism in Medicine (AIM) Initiative. In the Department of Medicine, Dr. Ripp serves as core faculty for the IM Residency Training Program and faculty in the Mount Sinai Visiting Doctors home-based primary care program. In addition, Dr. Ripp is the Co-founder and Co-Director of CHARM, the Collaborative for Healing and Renewal in Medicine, an international group of medical educators, academic medical center leaders, experts in burnout research and interventions, and learners all working to promote learner and trainee wellness. Dr. Ripp's primary research interest is in physician burnout and well-being, for which he has received grant support and has published and lectured widely. His multicenter studies have served to better elucidate the causes and consequences of physician burnout and have explored interventions designed to promote trainee well-being.

Craig Katz, MD is a Clinical Professor of Psychiatry, Medical Education, and System Design and Global Health at the Icahn School of Medicine at Mount Sinai, and the founder and director of Mount Sinai's Program in Global Mental Health. He organized the psychiatric response to 9/11 in New York City through an organization he co-founded, Disaster Psychiatry Outreach, including founding and directing the World Trade Center Mental Health Screening and Treatment Program for 9/11 responders. He has also been deeply involved in researching the mental health toll of child separation at Immigration and Customs Enforcement centers, showing the extent of mental health disorders in detained children. Dr. Katz holds numerous positions in the Department of Medical Education, including as a Faculty Advisor for medical students; Faculty Director of the mental health program within our student run, faculty facilitated primary care clinic, EHHOP; and Associate Director for Advocacy in the Mount Sinai Human Rights Program.

\section{Authors and Affiliations}

\section{Alexandra Saali ${ }^{1} \cdot$ Emma R. Stanislawski ${ }^{1,2}$ iD $\cdot$ Vedika Kumar $^{1,3} \cdot$ Chi Chan $^{4,5}$. Alicia Hurtado ${ }^{5}$ Robert H Pietrzak ${ }^{6,7}$. Dennis S. Charney ${ }^{5}$. Jonathan Ripp ${ }^{6}$. Craig L. Katz ${ }^{5}$}

1 Department of Medical Education, Icahn School of Medicine at Mount Sinai, New York, NY, USA

2 Department of Psychiatry, Maine Medical Center, Portland, ME, USA

3 Department of Psychiatry, Zucker Hillside Hospital, New York, NY, USA

4 Mental Illness Research Education and Clinical Center, James J. Peters VA Medical Center, Bronx, NY, USA

5 Department of Psychiatry, Icahn School of Medicine at Mount Sinai, New York, NY, USA

6 Department of Psychiatry, Yale University School of Medicine, New Haven, CT, USA

7 U.S. Department of Veterans Affairs National Center for Posttraumatic Stress Disorder, VA Connecticut Healthcare System, West Haven, CT, USA 